\title{
Pulmonary arteriovenous fistula by contrast- transthoracic echocardiography- a case report
}

\begin{abstract}
Contrast echocardiography with harmonic imaging and contrast injection has improved the accuracy and reproducibility compared to imaging without contrast. Therefore, while evaluation of capillary free communications between pulmonary and systemic circulation is deemed important, assessment after contrast injection should be the preferred approach. We report a case of 60years old male with alcoholic liver cirrhosis and pulmonary arteriovenous fistula diagnosed by contrast echocardiography. This revealed delayed appearance of contrast 4-5 cardiac cycles with large numbers and the same density of micro-bubbles in all four chambers after 10 cardiac cycles, and when the injection was stopped, there were micro-bubbles in the left cardiac chambers meanwhile none in the right cardiac chambers.
\end{abstract}

Keywords: contrast- transthoracic echocardiography, pulmonary arteriovenous malformation
Volume 8 Issue 6 - 2017

\begin{abstract}
Madhu Gupta,' Liping Chen,' Maheshwar Prasad, ${ }^{2}$ Rajesh Panjiyar, ${ }^{3}$ Sachin Dhungel, ${ }^{3}$ Shankar Laudari, ${ }^{3}$ Subramanyam G'

'Cardiovascular Disease Center, jilin University, China

${ }^{2}$ National Medical College, Tribhuvan University, Nepal ${ }^{3}$ Department of Cardiology, College of Medical Science, Kathmandu University, Nepal
\end{abstract}

Correspondence: Shankar Laudari, Department of cardiology. College of Medical Science, Kathmandu University, Bharatpur, Nepal,Email ishankar2@hotmail.com

Received: March 21, 2017| Published: May 16, 2017

\section{Case report}

A 60years old male, known case of diabetes mellitus, hypertension and alcoholic hepatic cirrhosis had exercise induced chest discomfot, shortness of breath, fatigue and lips cyanosis half a year ago, which relieved at rest. But later he became serious for one week and visited our department for further management. He had no cough, fever, dizziness, headache or bleeding history from any site. He had pituitary adenoma, which was surgically removed 6years ago. He denies history of chronic infections such as tuberculosis or actinomycosis or family history of hereditary hemorrhagic telangiectasis (HHT). Physical examination revealed cyanosis of the face and lips, clubbing, numerous spider naevi on neck, chest and palm and no telangiectasia on mucosa or fundi. His blood pressure was $130 / 80 \mathrm{mmHg}$, heart rate was 76 beats/min and resting oxygen saturation was $66 \%$ in room air. A grade 1/6 soft systolic murmur was heard on the upper left sternal border and clear lungs sound. There were no bruits. The hemoglobin was $11.5 \mathrm{gm} / \mathrm{dL}$ and the hematocrite was $35.5 \mathrm{gm} / \mathrm{dL}$. Arterial blood gas analysis showed Pao $264 \mathrm{mmHg}$ on room air and after $100 \%$ of oxygen $74 \mathrm{mmHg}$ with Paco2 $41 \mathrm{mmHg}$ and $\mathrm{PH}-7.35$. Chest radiography and electrocardiogram did not show any abnormalities. Pulmonary function test showed alveolar dispersion function severely reduced. Transthoracic echocardiography showed normal findings (No PFO/ ASD/VSD/PDA detected). We performed contrast echocardiography using agitated saline. A delayed appearance of a substantial amount of micro-bubbles in the left atrium greater than three cardiac cycles after appearance in the right atrium and ventricle (Figure 1) which was suggestive of pulmonary arteriovenous fistula. The delayed appearance and a large amount of micro-bubbles in the left atrium preclude the intracardiac shunting resulting from a patent foramen ovale (PFO) or arterial septal defect (ASD). Interestingly, the density of micro-bubbles was the same in the left and right cardiac chamber even after 10 cardiac cycles (Figure $2 \& 3$ ) and when the injection stopped, there were micro-bubbles in the left cardiac chambers meanwhile none in the right cardiac chambers (Figure 4). Because the hepatic function of the patient was in compensatory period, we gave him oxygen-inhalation and beta-blockers in order to decrease the oxygen demands and to lower the portal vein pressure. Moreover, he was advised to avoid sudden standing. The cyanosis improved, and the patient was discharged from the hospital and on regular follow-up. The patient was advised for pulmonary angiography but refused.

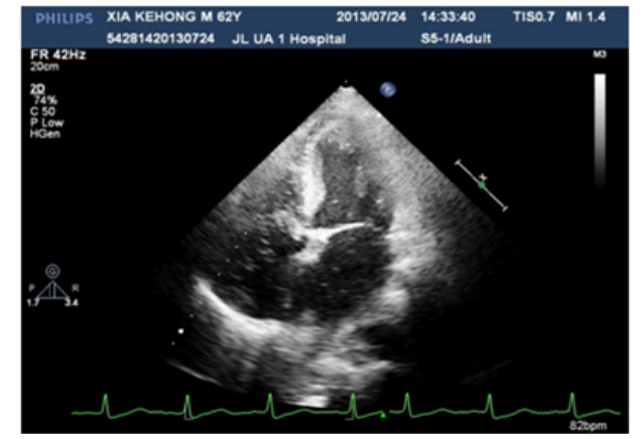

Figure I Micro-bubble appears in RA, RV, delayed appearance in LA, LV.
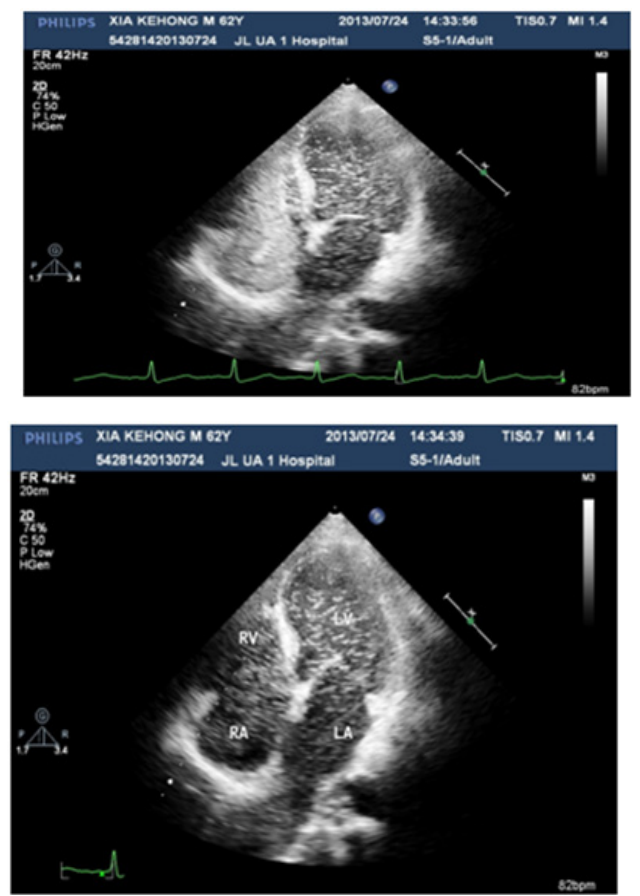

Figure $\mathbf{2}$ \& $\mathbf{3}$ The density of micro-bubbles was the same in the left and right cardiac chambers even after 10 cardiac cycles. 


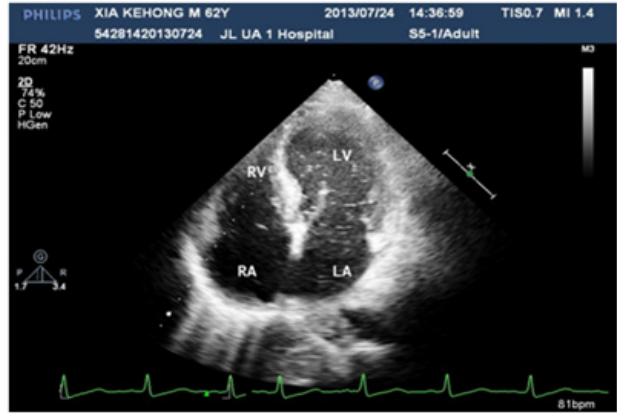

Figure 4 When the injection was stopped, there were micro-bubbles in the left cardiac chambers meanwhile none in the right cardiac chambers.

\section{Discussion}

Pulmonary arteriovenous malformations (PAVM) also known as pulmonary arteriovenous fistula is a rare cardiovascular disorder, first described at autopsy in $1897 .{ }^{1}$ Pulmonary arteriovenous malformations (PAVMs) are caused by abnormal communications between pulmonary arteries and pulmonary veins, which are most commonly congenital in nature. Although only $10 \%$ of the cases of pulmonary arteriovenous fistula are identified in infancy or childhood, with dyspnea on exertion, clubbing or cyanosis, however, the incidence gradually increases through the fifth and sixth decades of life. Approximately $70 \%$ of the cases of PAVM are associated with hereditary hemorrhagic telangiectasia (HHT), an autosomal dominant disorder. Conversely, about 15 to $35 \%$ of patients with HHT have PAVM. ${ }^{2}$ Abnormal communications between blood vessels of the lung may also be found in a variety of acquired conditions such as hepatic cirrhosis, schistosomiasis, tuberculosis, mitral stenosis, trauma, actinomycosis, Fanconi's syndrome and metastatic thyroid carcinoma. $^{3,4}$ In hepatic cirrhosis, hepatopulmonary syndrome (HPS) leads to the developments of pulmonary arteriovenous malformations are initially microscopic and progress to a large macroscopic malformations. Cyanosis, clubbing and arterial oxygen desaturation occur in patients with liver disease, attributed to the presence of pulmonary or peripheral arterio-venous shunting. ${ }^{5}$ Although the causes of hypoxemia in hepatic cirrhosis seem to be multi-factorial, the intrapulmonary arteriovenous shunt is the most important factor and contrast echocardiography is widely used most sensitive non-invasive screening test for the evaluation of pulmonary AVMs. Contrast echocardiography is considered the gold stander, ${ }^{6}$ for evaluating intracardiac shunts, because of its ability to provide excellent anatomic imaging of intracardiac structures as well as it shows contrast transition from the right to the left cardiac chambers. Intracardiac shunt lesions are characterized by the appearance of contrast material in the left atrium and ventricle within two cardiac cycles, whereas the appearance of contrast material in intrapulmonary right-to-left shunts shows a delay of 3 to 6 cardiac cycles. ${ }^{7}$ In our patient, contrast echocardiography showed that intact interatrial and interventricular septa along with the delay of contrast appearance of 4-5 cardiac cycles with large numbers and the same density of micro-bubbles in all four chambers after 10 cardiac cycles, and when the injection was stopped, there were micro-bubbles in the left cardiac chambers meanwhile none in the right cardiac chambers. This indicated the presence of an extracardiac intrapulmonary shunt. Although, pulmonary angiography is considered the gold standard for evaluating and localizing extra-cardiac shunt, there are many studies that hypothesized agitated saline contrast echocardiography is a useful screening test for pulmonary arteriovenous malformations. ${ }^{8}$ Moreover, the availability, simplicity and non-invasiveness of contrast echocardiography makes it a feasible screening test overall.

\section{Conclusion}

We describe a case of pulmonary arteriovenous fistula associated with alcoholic liver cirrhosis; diagnosed by contrast two-dimensional echocardiography. As contrast two-dimensional echocardiography is easily available, sensitive and non-invasive technique used for detecting intracardiac, intrathoracic and extracardiac shunts, the characteristic of contrast imaging can be a useful screening tool in cyanotic patients with liver diseases and in whom pulmonary atreriovenous fistula is suspected.

\section{Acknowledgments}

None.

\section{Conflicts of interest}

Author declares there are no conflicts of interest.

\section{Funding}

None.

\section{References}

1. Churton T. Multiple aneurysms of the pulmonary artery. $\mathrm{Br} \mathrm{Med} J$. 1897;1:1223-1225.

2. McAllister KA, Grogg KM, Johnson DW, et al. Endoglin, a TGFbeta binding protein of endothelial cells, is the gene for hereditary haemorrhagic telangiectasia type 1. Nat Genet. 1994;8(4):345-351.

3. Lange PA, Stoller JK. The hepatopulmonary syndrome. Ann Intern Med. 1995;122(7): 521-529.

4. Prager RL, Laws KH, Bender HW. Arteriovenous fistula of the lung. Ann Thoracic Surgery. 1983;36(2):231-239.

5. Keren G, BoichisH, Zwas TS,et al. Pulmonary arterio-venous fistulae in hepatic cirrhosis. Arch Dis Child. 1983;58(4):302-304.

6. Vedrinne JM, Duperret S, Bizollon T, et al. Comparison of transesophageal and transthoracic contrast echocardiography for detec-tion of an intrapulmonary shunt in liver disease. Chest. 1997;111(5):1236-1240.

7. Shub C, Tajik AJ, Seward JB. Detecting intrapulmonary right-toleft shunt with contrast echocardiography: Observations in a patient with diffuse pulmonary arteriovenous fistulas. Mayo ClinProc. 1976;51(2):81-84.

8. NanthakumarK, Graham AT, Robinson TI, et al. Contrast echocardiography for detection of hereditary hemorrhagic telangiectasia. Am Heart J. 2001;141(2):243-246. 